\title{
Study on Spatiotemporal Evolution Characteristics of Regional Annual Precipitation
}

\author{
Xianqi Zhang*(**)(***), Zhiwen Zheng*† and Rulin Ouyang**** \\ *School of Water Conservancy, North China University of Water Resources and Electric Power, Zhengzhou 450046, \\ China \\ **Collaborative Innovation Center of Water Resources Efficient Utilization and Protection Engineering, Zhengzhou \\ 450046, China \\ ***Technology Research Center of Water Conservancy and Marine Traffic Engineering, Henan Province, Zhengzhou \\ 450046, China \\ ****Water Resources Management Center, Ministry of Water Resources, Beijing 100038, China \\ $\dagger$ Corresponding author: Zhiwen Zheng; 604267813@qq.com
}

Nat. Env. \& Poll. Tech.

Website: www.neptjournal.com

Received: 23-12-2020

Revised: $21-07-2020$

Accepted: 04-03-2021

Key Words:

Innovation trend analysis (ITA)

Precipitation

Wavelet analysis

\begin{abstract}
The study of the temporal and spatial evolution of precipitation is of great importance for the efficient use of water resources. This paper examines the long series of precipitation in Henan province from 1959-2018. Innovative Trend Analysis (ITA) method and Mann-Kendall (MK) test were used to analyze the characteristics of precipitation trend changes. Mann-Kendall (MK) mutation test and the sliding $\mathrm{T}$ method were used to study the jump features of precipitation. Wavelet analysis of the cyclical characteristics of precipitation. The results show that the spatial distribution of precipitation in Henan Province is uneven, with a gradual increase from north to south. The precipitation of northern, central and southern regions showed a downward trend, while that of western regions showed an upward trend. Both the northern and southern regions experienced jump features in precipitation around 1975 and 2008, while the western region experienced jump features around 1962 and 1980 , and the central region experienced jump features around 1980; There are 3.5 different scales of "abundance and depletion" in the northern, western and southern regions, with a first principal cycle of $28 \mathrm{a}$, and 5.5 different scales of "abundance and depletion" in the central region, with a first principal cycle of $17 a$.
\end{abstract}

\section{INTRODUCTION}

With global warming, the hydrological cycle processes such as regional precipitation, evaporation and runoff have changed. The frequency of extreme weather and climate events raises widespread concerns about climate change. Rainfall is a major component of the water cycle and its variability is closely linked to droughts and floods, which can threaten water supply, agricultural irrigation and socio-economic development. The study of precipitation characteristics is therefore essential for flood control and drought prevention, rational use, optimal allocation and scientific management of water resources. (Zhou et al. 2020) analyzed precipitation trends and mutation characteristics in the North Canal basin from 1960-2016 using linear regression, Mann-Kendall mutation test, wavelet analysis and other methods. (Animashaun et al. 2020) Investigation of the temporal and spatial variability of rainfall in 33 micro-watersheds in the Central Hydrological Zone of Niger, Nigeria, from 1911-2015. (Wang et al. 2020) studied precipitation trends in the Yangtze River Delta from 1961 to 2016 using the Innovative Trend Analysis (ITA), Theil-Sen and Mann-Kendall methods, and verified the validity of the ITA method. (Chen et al. 2016) analyzed the trend and cyclical evolution of annual and seasonal precipitation from 1960-2006 at 49 meteorological stations in Liaoning province. At present, the evolution of precipitation is mostly studied in terms of trends and cycles in a single time series for an area or river basin, which does not sufficiently reflect the representativeness and spatial variability of regional precipitation evolution.

Henan Province is located in central China and covers a total area of $167000 \mathrm{~km}^{2}$, spanning four river basins: the Yangtze River, the Yellow River, the Huai River and the Hai River. The climate is characterized by a transition from subtropical to warm-temperate from south to north and from plain to hilly mountainous from east to west, and the precipitation is unevenly distributed over time and space.

As shown in Fig. 1, the daily measured precipitation data for the period 1959-2018 was selected from four weather 
stations in Anyang, Lushi, Zhengzhou and Xinyang. An analysis of 60-year precipitation trends in Henan Province using the Innovative Trends Analysis (ITA) and Mann-Kendall methods. The Mann-Kendall mutation test and the sliding $\mathrm{T}$ method for analyzing precipitation mutation characteristics. Analysis of its period using Morlet wavelets. The temporal and spatial characteristics and patterns of precipitation sequences are fully studied to provide reference for flood control and drought prevention, scientific management of water resources and agricultural production in Henan Province.

\section{DATA SOURCES}

In this paper, daily precipitation data for 60 years from 1959 to 2018 from four meteorological stations in Anyang (north), Lushi (west), Zhengzhou (central) and Xinyang (south), provided by the China Meteorological Data Network, are used. Daily precipitation data is collated into adult precipitation data for study.

\section{METHODS}

\section{Innovative Trends Analysis (ITA) Method}

There are a number of methods for studying trends in precipitation, such as the Mann-Kendall (MK) test and the
Spearman's rho (SR) test. These methods provide a better picture of precipitation trends, however, they require restrictive assumptions such as data length, normality, series independence, etc. (Alashan 2018). These features may sometimes be absent in the structure of the hydrological time series. ITA method is a trend analysis method that does not rely on restrictive assumptions and is highly effective (Alifujiang et al. 2020, Gedefaw et al. 2018, Huang et al. 2018, Li et al. 2019).

ITA is a graphical method for displaying hydrometeorological trends based on time series on a Cartesian coordinate system. The raw data is divided equally into two parts according to the time series, each part being arranged in ascending order. The top half of the data is defined as the first subsequence on the $\mathrm{x}$-axis and the bottom half as the second subsequence on the y-axis, as shown in Fig. 2. A 1:1 $\left(45^{\circ}\right)$ line represents a non-trend line and a scatter falling above (below) a 1:1 line represents an upward (downward) trend in the time series. A scatter falling within $5 \%$ of the $1: 1\left(45^{\circ}\right)$ straight line means that there is no significant trend in the time series. In this paper, rainfall is divided into three sub-areas: 'low', 'medium' and 'high' to detect trends in rainfall within the different sub-areas (Şen 2012, Şen 2017).

The slope $s$ of the innovative trend analysis method can be calculated according to the following equation.

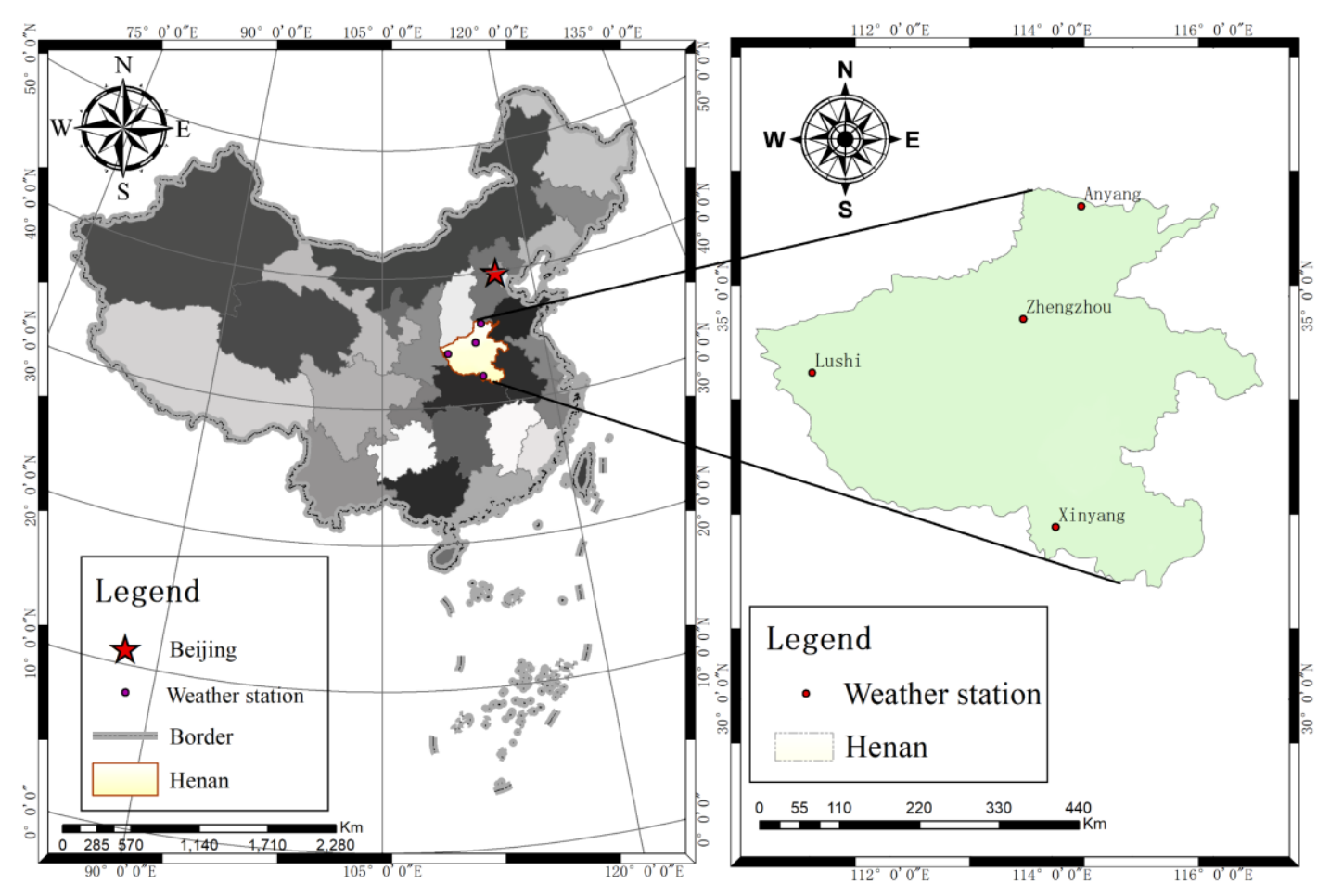

Fig. 1: Diagram of the study area. 


$$
s=\frac{2\left(\bar{y}_{2}-\bar{y}_{1}\right)}{n}
$$

Where: $n$ is the length of the sequence, $\bar{y}_{2}$ is the arithmetic mean of the vertical coordinates and $\bar{y}_{1}$ is the arithmetic mean of the horizontal coordinates.

\section{Mann-Kendall (MK) Test}

The MK test is a non-parametric trend detection method. The advantage is that the data is not required to be normally distributed and is rarely disturbed by outliers. (Liu et al. $2012 b)$. The basic formula is as follows:

$$
\begin{gathered}
S=\sum_{i=1}^{n-1} \sum_{j=i+1}^{n} \operatorname{sgn}\left(x_{j}-x_{i}\right) \\
\operatorname{sgn}\left(x_{j}-x_{i}\right)=\left\{\begin{array}{l}
+1, \text { if }\left(x_{j}-x_{i}\right)>0 \\
0, \text { if }\left(x_{j}-x_{i}\right)=0 \\
-1, \text { if }\left(x_{j}-x_{i}\right)<0
\end{array}\right.
\end{gathered}
$$

Where: $x_{j}, x_{i}$ denotes the sample data values and $j_{>} i$; $n$ denotes the length of the data series. When $n>10$, the $S$ distribution tends to be normal. The variance is calculated is as follows:

$$
\begin{gathered}
\operatorname{var}(S)=\left[n(n-1)(2 n+5)-\sum_{t} t(t-1)(2 t+5)\right] / 18 \\
Z=\left\{\begin{array}{cl}
\frac{S-1}{\sqrt{\operatorname{var}(S)},} & S>0 \\
0 & S=0 \\
\frac{S+1}{\sqrt{\operatorname{var}(S)},}, & S<0
\end{array}\right.
\end{gathered}
$$

In bilateral trend tests, for a given level of significance, the original hypothesis is considered unacceptable if $|Z| \geq Z_{1-\alpha / 2}$ That is, at a confidence level of $\alpha$, there is a clear upward or downward trend in the time series data. For a given level of significance, a is considered an unacceptable hypothesis. When $|z|$ is greater than or equal to the threshold value $Z_{1-\alpha / 2}=1.96,2.58$ it means that both an upward and downward trend is evident, passing the $95 \%$ and $99 \%$ level of significance test respectively (Guo et al. 2015, Liu et al. 2012a, Yu \& Chen 2013).

\section{Morlet Wavelets}

Wavelet analysis is a high-resolution analytical method that enables the decomposition of time series into the time and frequency domains in order to obtain the inherent evolution of the sequence. Its wavelet function is oscillatory and can quickly decay to zero (Heng 2003, Li et al. 2011).
For any function $f(t) \subset L^{2}(R)$, where $L^{2}(R)$ denotes the squared productive space and $R$ denotes the set of real numbers, the continuous wavelet transformation is:

$$
W_{f}(a, b)=\frac{1}{\sqrt{|a|}} \int_{-\infty}^{+\infty} f(t) \bar{\psi}\left(\frac{t-b}{a}\right) d t
$$

Where: $W_{f}(a, b)$ is the wavelet coefficient; $a$ is the periodic scale factor; $b$ is the time shift factor; $\bar{\psi}\left(\frac{t-b}{a}\right)$ is the complex conjugate function for $\psi\left(\frac{t-b}{a}\right)$.

Wavelet variance is a parameter reflecting the main time scale of the sequence, the value of which can be obtained by integrating the continuous wavelet transform coefficients, calculated by the following formula:

$$
\begin{gathered}
\operatorname{Var}(a)=\int_{-\infty}^{+\infty}\left|W_{f}(a, b)\right|^{2} d b \\
\psi(\mathrm{t})=\mathrm{e}^{w_{0} t i} e^{-t^{2} / t}
\end{gathered}
$$

\section{RESULTS AND DISCUSSION}

\section{Trend Analysis}

The trends in precipitation at the four stations in Anyang, Lushi, Zhengzhou and Xinyang are quantitatively analyzed using the MK test method and the Innovative Trend Analysis (ITA) method. The results of the calculations are shown in Table 1.

According to the calculations in Table 1, the annual precipitation MK values for Anyang, Zhengzhou and Xinyang are $-0.53,-1.73$ and -0.11 respectively, which are all less than zero and $\left|Z_{c}\right|<1.96$, indicating a non-significant downward trend. Whereas the annual precipitation MK for the Lushi is 1.05 greater than 0 but $\left|Z_{c}\right|<1.96$ with a non-significant upward trend. The Innovative Trend Analysis (ITA) method agrees with the results of the MK trend analysis. Precipitation in Anyang shows an upward trend in the 'low' zone and a downward trend in the 'medium' and 'high' zones. In combination with Fig. 2(a), there is a clear upward trend in precipitation for less than $300 \mathrm{~mm}$ and a downward trend for more than $650 \mathrm{~mm}$, but not significant. The precipitation trends in the 'low' and 'medium' divisions of Lushi are consistent

Table 1: Trend test calculation results

\begin{tabular}{|llllll|}
\hline Sites & $Z_{c}$ & \multicolumn{4}{c|}{$\mathrm{S}$} \\
\cline { 3 - 6 } & & Year & low & medium & High \\
\hline Anyang & -0.53 & -0.80 & 2.20 & -0.15 & -7.93 \\
Lushi & 1.05 & 0.17 & 0.65 & 1.43 & -25.62 \\
Zhengzhou & -1.73 & -0.04 & 3.14 & 0.42 & -25.21 \\
Xinyang & -0.11 & -1.06 & 0.29 & -3.94 & -9.60 \\
\hline
\end{tabular}


with the annual precipitation trends, with a non-significant upward trend, but with a significant downward trend in the 'high' divisions. In combination with Fig. 2(b), most of the precipitation in Lushi is concentrated in the $400-700 \mathrm{~mm}$ range and the trend is not significant, with a significant downward trend below the 5\% trend line for precipitation greater than $900 \mathrm{~mm}$. The trend of precipitation in the 'low' and 'medium' subdivisions of Zhengzhou is increasing but not significantly, while the downward trend of precipitation in the 'high' subdivisions is significant. According to Fig. 2(c), precipitation greater than $900 \mathrm{~mm}$ in Zhengzhou is below the $5 \%$ trend line and it shows a significant downward trend. According to Fig. 2(d), there is a significant downward trend in precipitation in Xinyang between 1300 and $1500 \mathrm{~mm}$.

Rainfall in Henan Province increases from north to south. Precipitation in the north and west-central regions is mostly

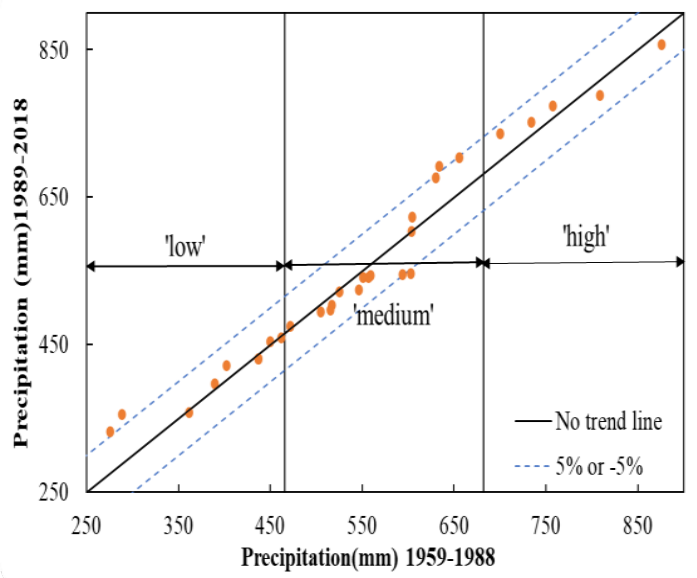

(a) Anyang

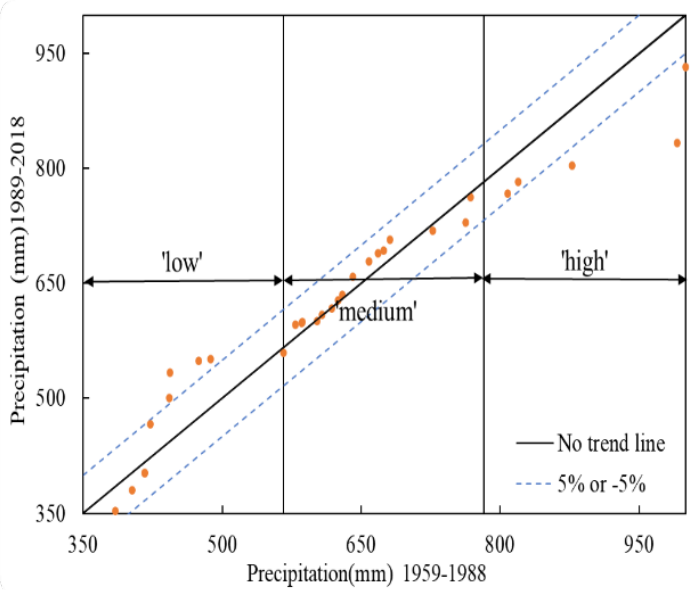

(c) Zhengzhou in the range of $300-1000 \mathrm{~mm}$, while in the south it is mostly in the range of $800-1600 \mathrm{~mm}$, with an uneven spatial distribution of precipitation. In the northern and south-central parts of Henan there is a downward trend in precipitation over the years and an upward trend in the west, but the change in trend is not significant.

\section{Mutational Analysis}

The Mann-Kendall mutation test and the sliding T-test were used to analyze the characteristics of the annual precipitation mutations in Henan Province.

The Mann-Kendall mutation test plot (Fig. 3) and the sliding T-test plot (Fig. 4) for the four stations in Henan were plotted using MATLAB.

As can be seen from the Mann-Kendall mutation test curve (Fig. 3a). The intersections of UF statistics and UB

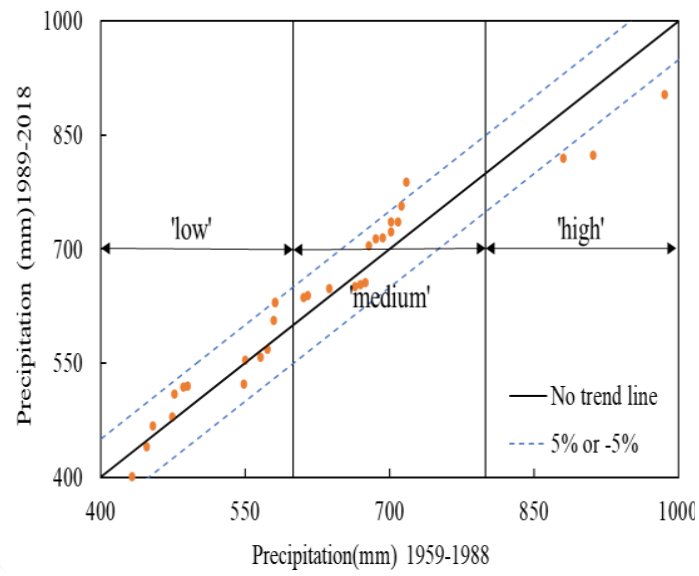

(b) Lushi

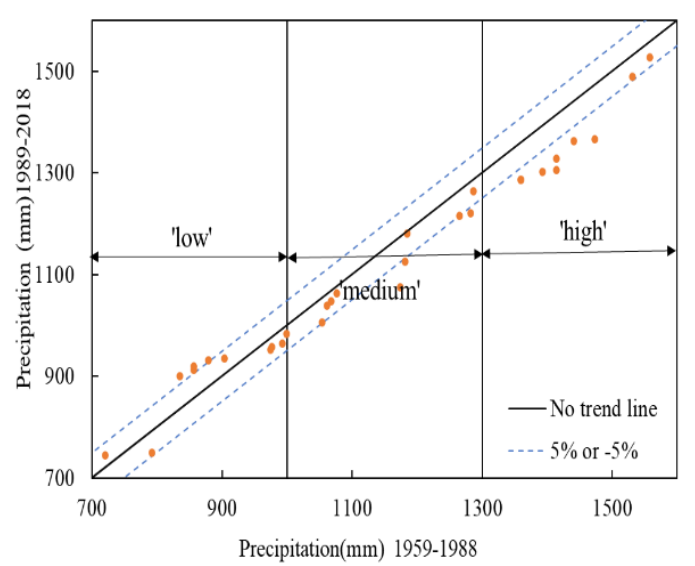

(d) Xinyang

Fig. 2: ITA precipitation trends at four stations in Henan. 
statistics are 1961, 1966, 1974, 2008 and 2017 respectively, indicating that the precipitation in Anyang was abruptly changed in 1961, 1966, 1974, 2008 and 2017.The UF statistics for the period 1965-2018 are all negative, indicating a decreasing trend in precipitation in Anyang. According to Fig. 3(b), the years in which the mutation occurred in Lushi were 1961, 1980, 1986, 2006 and 2013, and the UF statistic was negative in 1969-1981, indicating that there was a decreasing trend in precipitation in Lushi in 1969-1981 and an increasing trend in precipitation in Lushi in the remaining years. According to Fig. 3(c), Zhengzhou underwent mutations in 1961, 1980, 1985, 1995, 2011 and 2016, with UF statistics fluctuating up and down with no clear trend. According to Fig. 3(d), the years of the Xinyang mutation were 1963, 1973 and 1979.
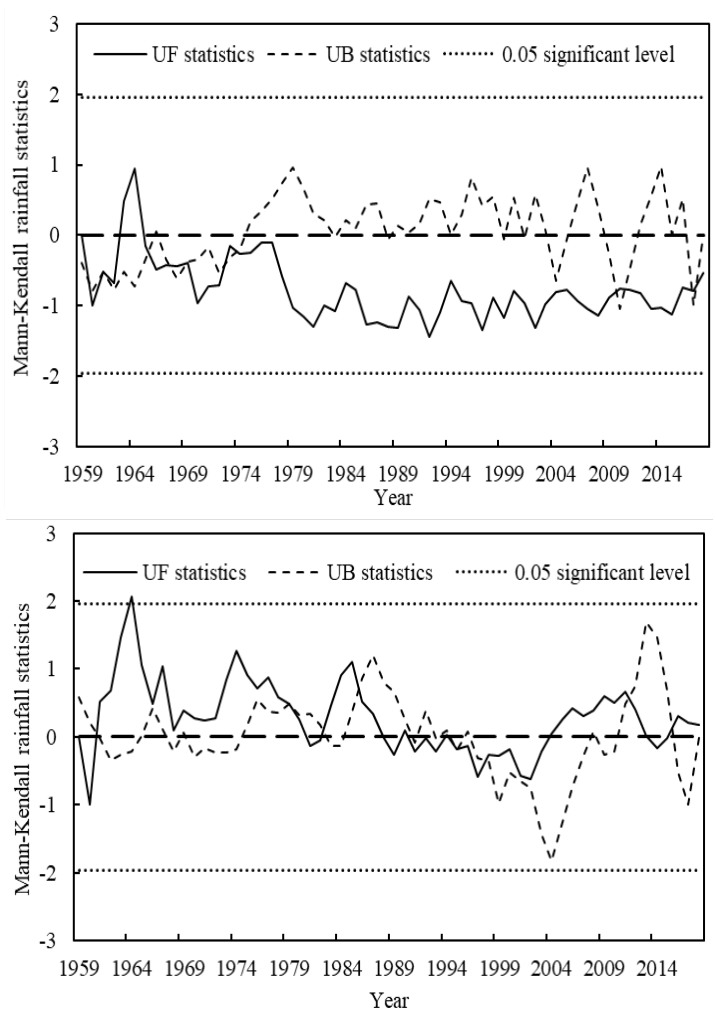

The annual precipitation at each station is subjected to a sliding T-test and the sliding T-test is plotted at the level of significance A (Fig. 4). As shown in Fig. 4(a), the Anyang mutation years are 1975, 1981 and 2008. See Fig. 4(b) for the year of the Lushi mutation, 1980, 1985. See Fig. 4(c) for the Zhengzhou mutation years of 1964, 1981, 1985 and 2002. As shown in Fig. 4(d), the year of the Xinyang mutation is 2008.

Combining the results of the Mann-Kendall mutation test and the sliding T-test plots, which are shown in Table 2, it was determined that precipitation in Anyang and Xinyang was mutated around 1975 and 2008, in Lushi around 1962 and 1980, and in Zhengzhou around 1980.

\section{Cyclical Analysis}

The cumulative anomaly of 60 years of sequential annual
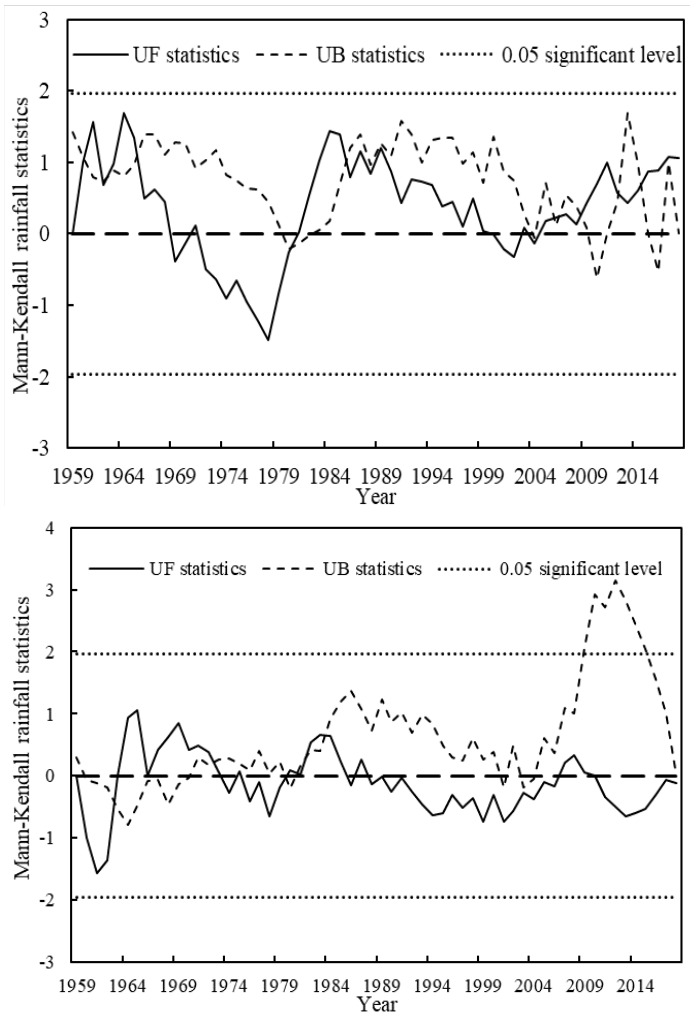

Fig. 3: Mann-Kendall mutation test curve.

Table 2: Rainfall mutation year

\begin{tabular}{|llll|}
\hline & Anyang & Lushi & Zhengzhou \\
\hline $\begin{array}{l}\text { Mann-Kendall mutation } \\
\text { test results }\end{array}$ & $1961,1966,1974,2008$, & $1961,1980,1986,2006$, & $1961,1980,1985,1995,2011,2016$ \\
$\begin{array}{l}\text { Sliding T mutation test } \\
\text { results }\end{array}$ & $1975,1981,2008$ & 2013 & \\
Mutation year & 1975,2008 & 1980,1985 & $1964,1981,1985,2002$ \\
\hline
\end{tabular}



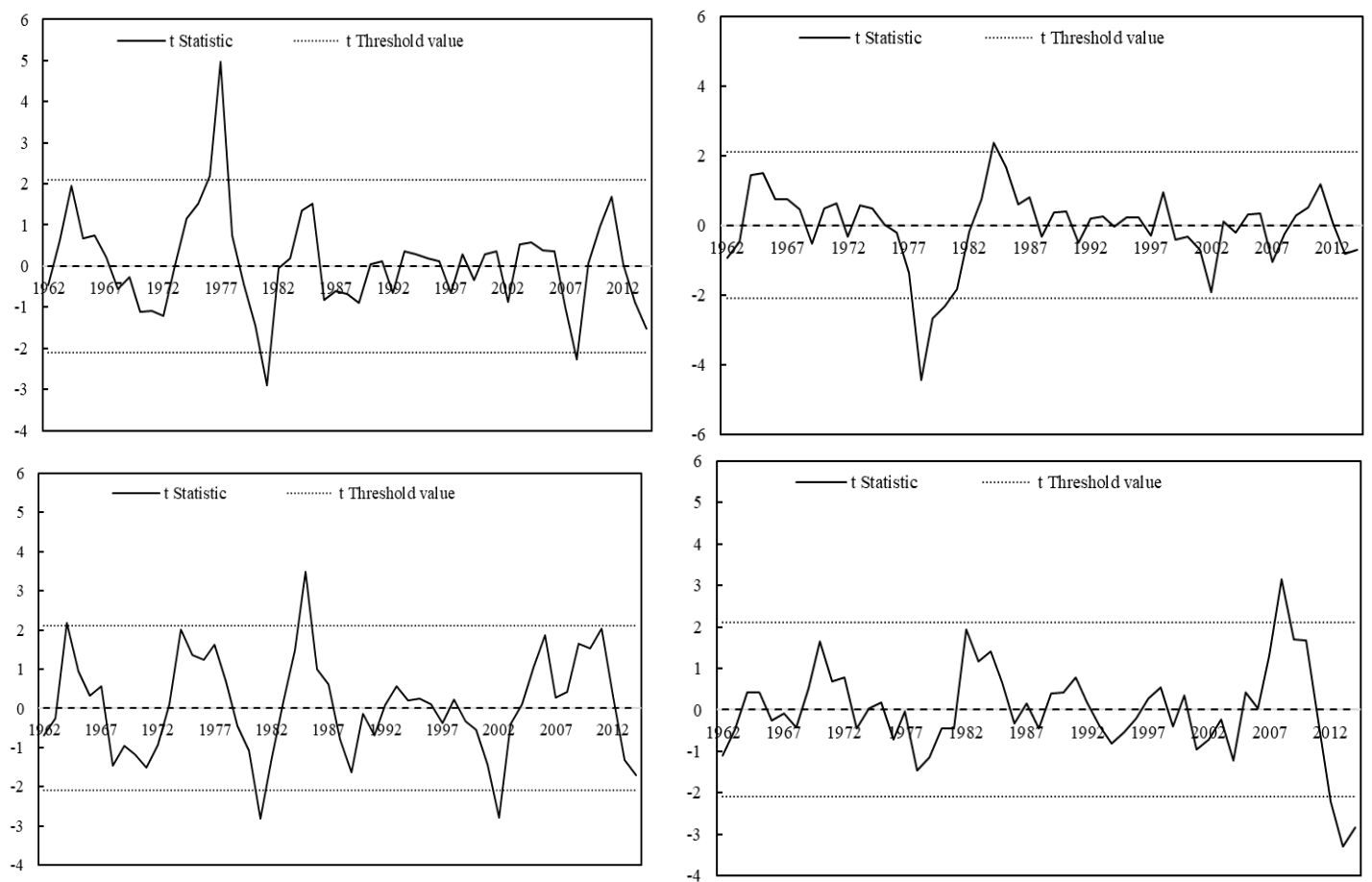

Fig. 4: Sliding T-test curve

precipitation data in Henan was processed, and Kolmogorov-Smirnov test was conducted on the annual precipitation data of four stations in Henan by SPSS software. The results showed that the annual precipitation data of the four stations in Henan presented an approximate normal distribution. Therefore, the MATLAB program is used to perform a wavelet transform on the series data, calculate the Modulus and real part of wavelet transform, and draw the wavelet variance plot of the annual precipitation of Henan 4 stations from 1959 to 2018 and the contour plot of the real part of wavelet, as shown in Fig. 5 and 6 respectively.

In the wavelet variance plot of annual precipitation at four stations in Henan from 1959-2018 (Fig. 5), the larger the variance value, the stronger the cyclical oscillation on the corresponding time scale. From Fig. 5(a), it can be seen that there are three peaks in the variance curve of annual precipitation in Anyang city, corresponding to time scales of 28a, 23a and 8a, respectively. This indicates that the oscillation cycles of the main flood precipitation in the northern region are 28a, 23a and 8a, with the largest peak on the 28a time scale and the strongest oscillation cycle being the first main cycle, and the second and third main cycles corresponding to the $23 \mathrm{a}$ and $8 \mathrm{a}$ time scales in turn. In combination with Fig. 6(a), the first main cycle 28a precipitation in Anyang experienced 3.5 alternations of abundance and depletion.
The fact that the 2018 precipitation wavelet contours are not closed and are roughly in the center of the solid part of the line suggests that the region will remain in an abundance state for the next 5 years.

The analysis in Fig. 5(b)-(d) is the same, with three peaks in Lushi, Zhengzhou and Xinyang, the first main period in Lushi being 28a and the second and third main periods corresponding to time scales $17 \mathrm{a}$ and $8 \mathrm{a}$, the first main period in Zhengzhou being 18a and the second and third main periods corresponding to time scales $28 \mathrm{a}$ and $8 \mathrm{a}$, and the first main period in Xinyang being $28 \mathrm{a}$ and the second and third main periods corresponding to time scales $18 \mathrm{a}$ and $8 \mathrm{a}$. According to Fig. 6(b)-(d), the first principal cycle of 28a rainfall in Lushi experienced 3.5 alternations of abundance and depletion, the first principal cycle of 17a rainfall in Zhengzhou experienced 5.5 alternations of abundance and depletion, and the first principal cycle of 28a rainfall in Xinyang experienced 3.5 alternations of abundance and depletion. In summary, Anyang, Lushi and Xinyang all have a first principal cycle of $28 \mathrm{a}$ and experience 3.5 alternate periods of abundance and dryness in their rainfall.

\section{CONCLUSIONS}

(1) Precipitation in Henan Province is in the range of $300-800 \mathrm{~mm}$ in the north and west-central regions, and 

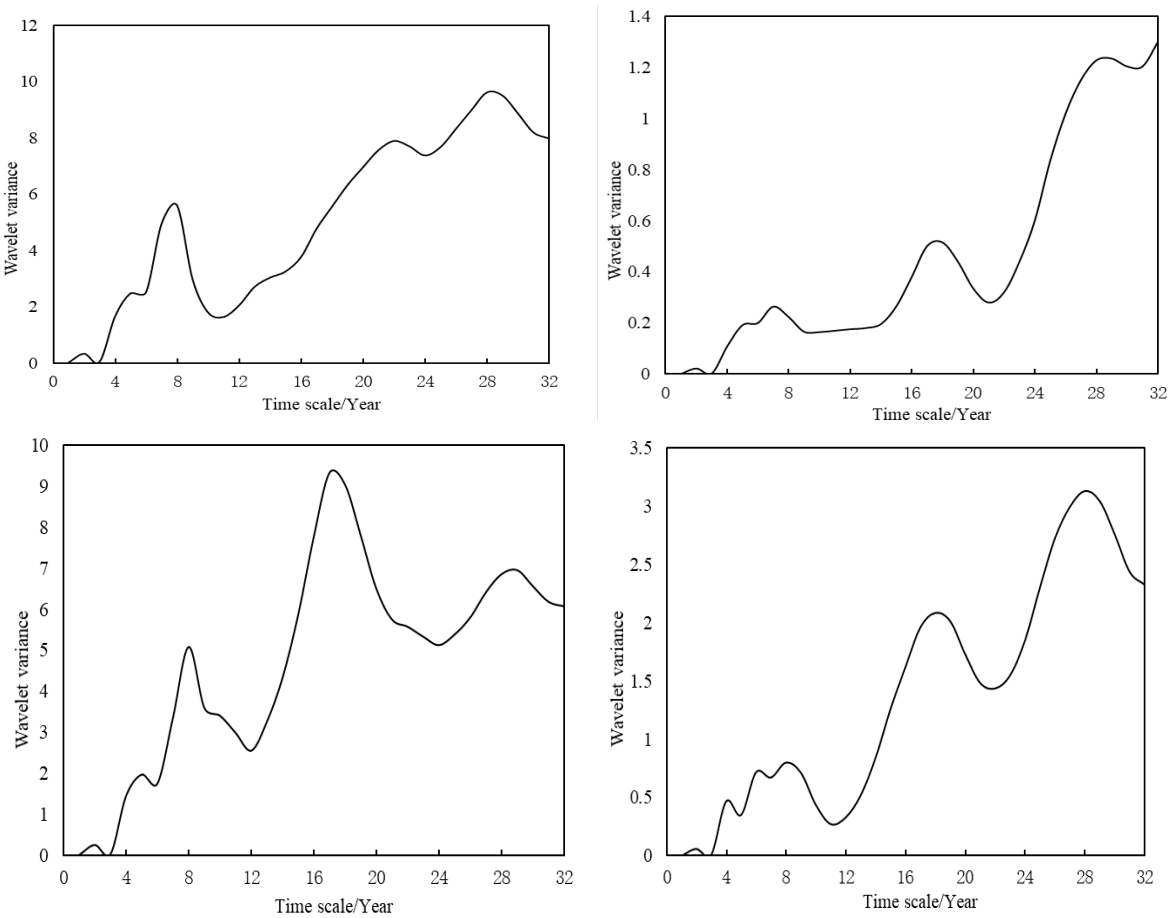

Fig. 5: Wavelet variance plot of annual precipitation at four stations in Henan, 1959-2018.
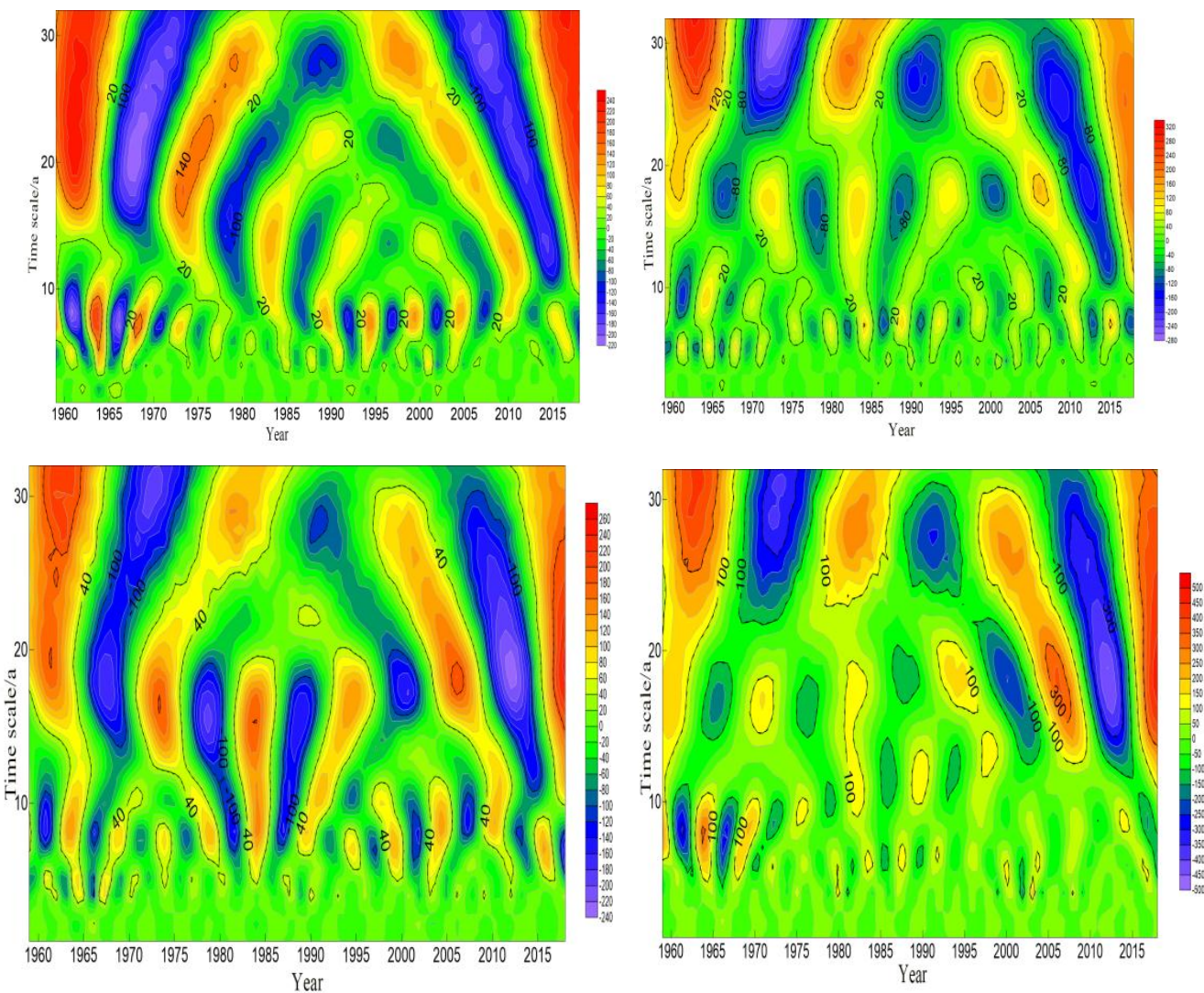

Fig. 6: Wavelet contours of annual precipitation at four stations in Henan, 1959-2018. 
$800-1500 \mathrm{~mm}$ in the southern regions, with the overall manifestation being a gradual increase in annual precipitation from north to south and west to east, and a gradual increase in precipitation intensity. The northern, central and southern regions of Henan show a non-significant downward trend in precipitation over the last 60 years, while the western regions show an upward trend, but the trend is not obvious. High-intensity precipitation in all regions shows a significant decreasing trend.

(2) Precipitation in the north and south of Henan Province was mutated around 1975 and 2008, in the west around 1962 and 1980, and in the central areas around 1980.

(3) Multiscale analysis of annual precipitation time series in Henan Province using Morlet wavelet analysis. There are three main cycles of annual rainfall in the northern, central and southern regions of Henan Province, with the first main cycle of 28 a experiencing 3.5 alternations of abundance and dryness, and the first main cycle of $17 \mathrm{a}$ in the central region experiencing 5.5 alternations of abundance and dryness, and the annual rainfall in each region will remain in an abundance state in the next $5 \mathrm{a}$.

(4) The evolution of regional precipitation is influenced by a variety of factors, but in this paper we only use mathematical methods to mine information within the 60 years of statistics themselves to study their evolutionary characteristics.

\section{REFERENCES}

Alashan, Sadik 2018. An improved version of innovative trend analyses. Arab. J. Geosci., 11(3): 50.
Alifujiang, Y., Abuduwaili, J., Maihemuti, B., Emin, B. and Groll, M. 2020. Innovative trend analysis of precipitation in the Lake Issyk-Kul Basin, Kyrgyzstan. J. Atmos., 11(4).

Animashaun, I.M., Oguntunde, P.G., Akinwumiju, A.S. and Olubanjo, O.O. 2020. Rainfall analysis over the Niger central hydrological area, Nigeria: variability, trend, and change point detection. J. Sci. Afr., 8 .

Chen, T., Xia, G., Wilson, L.T., Chen, W., Chi, D. and Dietrich, S. 2016. Trend and cycle analysis of annual and seasonal precipitation in Liaoning, China. J. Adv. Meteorol., 2016.

Gedefaw, M., Yan, D., Wang, H., Qin, T., Girma, A., Abiyu, A. and Batsuren, D. 2018. Innovative trend analysis of annual and seasonal rainfall variability in Amhara regional state, Ethiop. J. Atmos., 9(9).

Guo, S., Guo, B., Wang, G. and Xiao, Q. 2015. Mann-Kendall method based water-sediment trend analysis of the upper Han River. J. Water Resour. Power, 33(11): 140-142.

Heng, T. 2003. Research on wavelet analysis and its applications. PhD thesis, Sichuan University.

Huang, D., Liu, Z. and Jiang, Q. 2018. Applying non-parametric MK and ITA methods to characterise changes in groundwater quality parameters - an example of the New Three Mine Aquifer. J. Water Resour. Water Engineering, 29(03): 7-13.

Li, J., Wu, W., Ye, X., Jiang, H., Gan, R., Wu, H., He, J. and Jiang, Y. 2019. Innovative trend analysis of main agriculture natural hazards in China during 1989-2014. J. Nat. Hazards, 95(3).

Li, M., Xia, J., Chen, S. and Meng, D. 2011. Wavelet analysis of precipitation changes in Beijing over the last 300 years. J. N. Resour., 26(06): 1001-1011.

Liu, Y., Zhuo, X. and Qing, A. 2012. Mann-Kendall analysis of precipitation trends in the Guanzhong Basin. Yellow River, 34(02): 28-30+33.

en, Z. 2012. Innovative trend analysis methodology. J. Hydrol Eng., 17(9).

en, Z. 2017. Innovative trend significance test and applications. Theor. Appl. Climatol., 127(3-4).

Wang, Y., Xu, Y., Tabari, H., Wang, J., Wang, Q., Song, S. and Hu, Z. 2020. Innovative trend analysis of annual and seasonal rainfall in the Yangtze River Delta, eastern China. Atmos. Res., 231.

Yu, Y. and Chen, X. 2013. Mann-Kendall based process delineation of runoff abundance change. J. Water Res. Water Eng., 24(01): 60-63.

Zhou, J., Wang, S., Liu, C., Han, Z., Du, Y. and Jiang, Z. 2020. Analysis of precipitation trends and abrupt changes in the last 57a in the North Canal basin. Hydropower Technol., 51(S1): 21-29. 